ingrowths and outgrowths. The other highly plastic element of the ovary is the theca interna cell, which transforms from a fibroblastic cell into an epithelioid secreting cell, luteinizes to become-very likely-the only functional endocrine element of the corpus luteum, and finally can become transformed back into a fibroblastic structure.

Dr. Henri Bayle (Paris) discussed spermatophagia in the human epididymis in cases of excurrent duct obstruction and showed slides of spermatorrhagerupture of the epididymal tubules through excessive internal pressure, with outflow of spermatozoa into the peritubular spaces, leading to obliteration of the epididymal canal. He then demonstrated his film showing the operative technique of vaso-epididymostomy; the beauty and lucidity of this film are beyond description. Dr. Bayle does not perform testicular biopsy first, but prefers to incise the head of the epididymis after having mobilized the testicle ; if moving spermatozoa are seen in the epididymal fluid, he proceeds with the operation, provided patency of the vas deferens can be demonstrated. $\mathrm{He}$ uses seven sutures of fine tantalum wire to anastomose the vas to the epididymis. Delicacy of touch and the avoidance of trauma are the prime requisites of success in this difficult operation. Out of 143 cases explored surgically, anastomosis was found impossible in thirty cases, and in a further six congenital absence of the vas was discovered. Vaso-epididymostomy was performed bilaterally in sixty cases, and unilaterally in forty-five; in a further four cases anastomosis of the vas deferens direct to the testicles was attempted. Of sixty-five surgically satisfactory cases, the azoospermia was cured in forty-five (66 per cent), twenty of these men producing in all thirty-two children. This rate of success, of course, is far better than any other surgeon has achieved.

The morning of June 28 was devoted to a symposium on spermatozoan morphology; this was opened by Prof. L. Harvey (Exeter), who gave a review of spermatogenesis and spermateliosis. The National Institute for Medical Research team of workers (C. F. Challice, P. E. Lake, E. J. C. Polge and J. Smiles) reported ultra-violet microscopy studies on living fowl germ cells and electronmicroscope studies of mouse and rabbit spermatozoa. They showed how the Golgi zone of the fowl spermatid developed into the acrosome. The tail filament was found to be motile from the earliest stage, the tail granule being the source of motility. Chromosome material aggregates, becoming a homogeneous mass within the nuclear membrane. It then elongates, later to shrink, leaving behind a free portion of the nuclear membrane, thus forming the manchette which later forms a rigid tube around the first part of the tail. Considerable advances in our knowledge of the morphology of rabbit and mouse spermatozoa have been afforded by the technique developed by these workers of cutting sections $0.2 \mu$ thick followed by electron microscopy. There is strong evidence for a double spiral structure of the mid-piece and tail around the axial filament, all within a fine outer membrane. The axial filament contains nine fibrils arranged circularly around a central one, and there appears to be a flange running lengthwise down the tail.

Mr. Geoffrey Grigg (Cambridge) also contributed electron-microscope studies on fowl spermatozoa, his findings being mainly in agreement with those of the previous speakers. The apical cap dissolves in distilled water, leaving the apical spine partly embedded in the head. He has regularly found eleven fibrils in the axial filament- two central and nine outer. There seems to be an inner core to each fibril which is more elastic and more resistant to tryptic digestion than the surrounding material. Dr. Bayle showed electronmicroscope photographs of human spermatozoa. Dr. John McLeod (United States) discussed spermatozoan morphology in relation to human fertility and, as a result of his large-scale studies, he has come to the conclusion that little correlation between sperm morphology and fertility can be discovered in the human. Poor morphology is commonly associated with poor motility - which has a very definite bearing on fertility-but otherwise, provided the sperm density exceeds 20 millions per $\mathrm{ml}$. and the percentage of active cells is not much below 40, fertility seems to depend mainly upon the quality of sperm motion.

Dr. John Wilkinson (Exeter) described the effects of aspermy virus upon nuclear behaviour in solanaceous plants. In the pollen and embryo sac mother cells; the pachytene threads become disorganized in the prophase of meiosis and the nucleoli are multiplied. Some of the chromosomes fail to pair, leaving behind univalents at metaphase and anaphase. This probably accounts for the 25 per cent diminution in seed production shown by affected plants. It was suggested that a competition may exist between the virus and the chromonematal material of the chromosomes for ribose-nueleic acid. Mrs. C. Harvey (Exeter) said she had made similar observations on testicular biopsy material from subfertile men, having also noted collapse of the chromonemata at prophase and loose and lagging chromosomes at metaphase and anaphase. This seemed to suggest a possible viral etiology for some cases of human male infertility.

In the last two papers, Dr. F. X. Gassner (United States) showed that the rate of fructolysis in seminal fluid is better correlated with the breeding efficiency of bulls than the conventional criteria of seminal analysis, and Dr. E. W. Macmillan (Liverpool) described the vascular pattern of the epididymis in the rat and the sequence of changes in the epididymis and testis following interruption of the superior epididymal and accessory capital arteries; the vasa efferentia became packed with spermatozoa, which led to their gross enlargement and to severe degenerative changes within the testis, while the caput epididymis underwent necrobiosis followed by obliterative fibrosis.

\section{EMULSIONS AND FILMS SYMPOSIUM AT SHEFFIELD}

$T$ HE British Society of Rheology organized a symposium on "Emulsions and Films" which was held in the University of Sheffield during March 28-29. All the papers were followed by discussion, and on the second day the meeting visited the chemistry laboratories of the University and inspected the apparatus used by the speakers at the meeting.

In the first paper, Dr. A. S. C. Lawrence pointed out that emulsions are always placed in a separate category of colloids as liquid-in-liquid systems-a classification to which objection can be made on two grounds : the fact that the liquid state of the disperse phase makes possible dispersion by shaking is of no fundamental importance (it is not even true for 
dispersions of true colloidal size, that is, oil hydrosols which are typical hydrophobic systems); and it has distracted attention from the really basic criteria-namely, that, although hydrophobic, very high concentrations ( 74 per cent) of the disperse phase are easily attained, and the particle size is much larger than in all other hydrophobic colloidal solutions. Emulsions are not, strictly, colloids at all ; they are opaque, and sedimentation, as creaming, is one of their most distinctive properties. They belong, in fact, to the neglected class of protected colloids.

It has become accepted that the stability of emulsions is due to the lowering of the free surface energy of the system by adsorption of surface-active emulsifying agent and that interfacial tension is a 'coagulating tendency'. This error of identifying the potential reduction of free energy with the driving force causing the reduction is one against which students learning thermodynamics are warned year after year. In any case, a lowering of the free surface energy can reduce only potential instability and cannot give an absolute stability : even if this thermodynamic quantity were the driving force in coagulation, reduction to zero would be required to give absolute stability. This, however, is the condition for miscibility of the two phases, and it is also a characteristic of emulsions that there is some residual interfacial surface tension which is responsible for the spherical shape of the droplets of the disperse phase. The emulsifying agent should be regarded as an energy barrier which is needed, as in all hydrophobic systems, to prevent aggregation or coalescence of particles subsequent to collisions due to their Brownian motion. The action of the energy barrier is to prevent coalescence following collision; it has no effect upon collision frequency. The mean Brownian velocity depends upon $T$ and inversely upon $n$-that is, the viscosity of the continuous phase and not that of the whole emulsion, which is always larger, usually by a large factor. Collision frequency will be highly dependent upon concentration obviously as a function of the cube root of it, since it is dependent upon the mean distance of separation of the droplets, all other factors being the same. Creaming will always increase collision frequency because it reduces the mean separation distance. For fine dispersions, Brownian motion is the rate-determining factor in the collision process but, as particle size increases, sedimentation becomes increasingly the rate-determining factor. Every chemist has had the opportunity of seeing this transition every time he has done an ether extraction. This kinetic picture must be extended because there will be a Maxwell distribution of Brownian velocities, and the number with sufficient energy to overcome the energy barrier to coalescence, $E$, will be given by the usual $n / n_{0}=\exp (-E / R T)$. 'Stability' means that $n / n_{0}$ is vanishingly small.

Surface tension has no place in this scheme; it determines the work to be done in dividing the disperse phase; it has nothing to do with the stability of emulsions. Adsorption of the emulsifying agent to the interface requires that it must lower the interfacial tension, but this lowering need not be large. It could be said that surface tension is involved in the coalescence stage in breaking: since, however, the first step in coalescence must involve an increase of the surface area of two spherical drops, it would appear that high interfacial tension will resist this step while a low value favours it and, therefore, instability - again, this is the opposite to the accepted view that low interfacial tension favours stability.
The energy barrier provided by the emulsifying layer may be electrical, mechanical or due to surface tension. The last-named class consists of powders acting by a differential wetting-angle with the two phases. Amphipathic substances which are also ionized may be good emulsifiers but some are not. It therefore seems that some other property is concerned, and Dr. Lawrence suggested that this is viscosity; either a high two-dimensional $\eta$ or a plasticity. It is likely, but not proved, that objection may be made against rigidity in the sense that, when such a layer is fractured, it cannot spread again to close a gap. Dr. Lawrence's emulsions of sea water in fuel oil are a case in which very high surface viscosity, without a charge and with very slight surface activity, is responsible for stability.

The work carried out in Sheffield which Dr. Lawrence reported to the meeting is based upon the following idea. The viscosity of the continuous medium may be increased and therefore reduce $\xi$ and, therefore, collision frequency, but this is only a reduction: it cannot give absolute stability in a liquid system. The high and anomalous viscosity of an emulsion of 50 per cent or more oil in soap solution is an indication of the surface viscosity of the emulsifying layer : laminar flow requires droplets to slip between their neighbours and, to do so, they must be distorted into ellipsoids; if, after passing, they relax to spheres before the next passage, the work used in deforming them will be dissipated as a micro-turbulence of the liquid medium associated with the flow of the droplet, and non-Newtonian flow will result. One immediate result is that the extent of the anomalous viscosity will be proportional to the surface area of the drops; that is, inversely proportional to their diameter. Direct measurements have been made of the surface viscosity and plasticity of solutions of soaps with various polar groups.

Other researches in progress at Sheffield are an investigation of the kinetics of coagulation using micro-photographic recording with phase-contrast objectives-no method exists for assessing the stability of exulsions other than counts of particle size. Some less laborious method is urgently needed and is under: consideration. $\zeta$ potentials are also being studied, and a drop-profile apparatus is being used for measuring interfacial tensions, especially when small. The work is being extended to the action of complexes of soap plus a molecule containing a polar group such as carboxyl, hydroxyl or amine.

The present views upon which the work is proceeding are fourfold : that best emulsifying efficiency requires the closest packing of the soap molecules at the interface; that soaps alone are not the best emulsifiers (carboxylates are an apparent exception because the acid soap is the useful agent); that closest packing and maximum efficiency are provided by complexes of soaps (of all sorts) and molecules containing a hydrocarbon chain of not less than six normal carbon atoms and a polar group such as hydroxyl, carboxyl or amine; and that these complexes, at sufficient concentration in bulk, are all liquid crystalline of the smectic type.

Mr. E. Rothwell (University of Sheffield and Imperial Chemical Industries, Ltd., Ardear) reviewed the work of Hatschek and Sibree and the later empirical relations between $\eta$ and concentration proposed by Richardson, Broughton and Squires and by Goodeve. He then mentioned the work of Rossi, Lyttleton and Traxler and of Richardson upon the effect of particle size upon $\eta$. He described his own 
experimental work with particular reference to the effects of the packing-density in the emulsifying layer, to the effect of particle size and to the composition of the soap adsorbed at the interface. $\eta$ was inversely proportional to particle size diameter for $a$ given physical state of the emulsifier layer. For a given particle size viscosity dècreases with increase of area per molecule adsorbed-that is, viscosity increases with increase of packing density, and therefore with fall of interfacial tension. This proves that the work done in flow in deforming droplets is not work done against $\gamma$, since that would require $\eta$ to vary with $\gamma$ in the opposite sense to that found, and the work must therefore be done against a surface viscosity, plasticity or elasticity. In agreement with Hatschek, it was found that, at very low rates of shear, $\eta$ is independent of rate of shear corresponding with a complete relaxation of droplets between successive distortion; $\eta$ then falls with increase of rate of shear but becomes constant again at high values corresponding with permanent distortion during flow.

The next speaker, Mr. B. C. Blakey (University of Sheffield), described measurements of the surface viscosity of soap solutions by the oscillating bob method. Sodium carboxylates, sodium alkyl sulphates and kationic soaps all have a high surface viscosity which is non-Newtonian except in the $n$-alkyl sulphates: the kationic soaps developed surface viscosity only on standing. The minimum concentration for appearance of surface viscosity is well below the critical concentration : in the carboxylate series, $\eta$ rises with increase of chain length at $20^{\circ} \mathrm{C}$. Sodium soaps have a higher value than that for potassium compounds. 'The effect is very large; for solutions of sodium cetyl sulphate the viscous drag on the bob due to the surface viscosity is equivalent to an immersion in water of the bob of $2-5 \mathrm{in}$.

The greatest care needs to be taken to exclude impurities even in very minute concentrations, and fractionation by frothing is especially valuable as a check. The addition of fatty acid to a carboxylate soap causes surface viscosity to appear at lower concentration than for the soap alone ; but alkali has the opposite effect, which suggests that the surface species is acid soap. Alkyl trimethylammonium soaps showed a pronounced maximum at the critical concentration, the most obvious explanation of which was impurity which was solubilized at the higher concentrations. This has since been confirmed. A solution sufficiently pure to show no minimum in the surface tension concentration curve did give a maximum in the surface viscosity curve, and surface viscosity is therefore a more sensitive method for detection of small amounts of impurity than is surface tension. The sodium alkyl sulphates show Newtonian surface and interfacial viscosities when pure, but anomalous when not rigorously purified.

Work on solubilization of substances such as alcohols and fatty acids upon soaps was described by Mr. A. J. Hyde (University of Sheffield). This differs from solubilization of hydrocarbons in two fundamental respects : the inert substance in excess passes to emulsion, the polar addition, as soon as excess is added, separates with the soap as a liquid layer, the other being water, and the soap additive complex becomes oleophilic; at sufficient concentration and with a longer hydrocarbon chain in the additive, liquid crystals of the smectic layer type are always formed. The texture of the smectic phase varies with the composition giving either anisotropic spherulites, Dupin's cyclides or myelinic figures. The last are usually of the normal curved form, but occasionally have sharp angles as if simulating crystals. The general scheme seems to be :

spherical micelle in soap solution + additive $\rightarrow$ smectic layer complex

With less hydrophobic additive, for example, benzylalcohol, considerable further lowering of surface tension occurs, paralleled by charges of electrical conductivity. This lowering of surface tension must mean closer packing in the adsorbed film. With these more hydrophilic complexes, emulsification does not take place.

- In a theoretical paper on the rheological behaviour of an emulsion, Dr. J. G. Oldroyd (Research Laboratory, Courtaulds, Ltd., Maidenhead) described how a finite interfacial tension between the two phases must give rise to elastic as well as viscous properties, since it provides a force tending to restore each deformed droplet of the disperse phase to its original spherical form. On the assumption that the droplet size is sufficiently small and uniform, an equation of state for a dilute emulsion subjected to small rates of strain was derived in the form of a linear relation between stress, rate of change of stress, rate of strain, and rate of change of rate of strain. The three constants in the equation were expressed in terms of the viscosities of the two phases, the interfacial tension and the volume concentration. The predicted effect of introducing a stabilizer would be principally to increase the relaxation time and retardation time of the system (by decreasing the interfacial tension) without changing the viscosity. But if hydrodynamic slipping were possible at the droplet boundaries due to the presence of a film of stabilizer, all three constants would be affected; preliminary calculations showed that it might be very difficult to detect slipping of this kind experimentally.

\section{A. S. C. LAWRENCE}

\section{SECOND SEMINAR FOR EUROPEAN SANITARY ENGINEERS}

$T$ $\mathrm{HE}$ second seminar for European sanitary engineers, sponsored by the Italian Government, the Rockefeller Foundation and the United Nations World Health Organization, was held in Rome during November 1951 ; representatives from sixteen European countries attended. The report of this meeting, published by the World Health Organization*, contains a great deal of information, which has not hitherto been easily available, on sanitary conditions on the Continent.

There was general agreement that there should be closer collaboration between sanitary engineers in different European countries. Many rivers, for example, flow through more than one country before reaching the sea, and it is clearly desirable that the discharge to them of polluting substances should be controlled in the common interest. The problems which arise in this kind of international planning are, of course, partly political, but are more likely to be solved if there is general agreement on the technical issues involved. At present the importance of the

* World Health Organization. Second Seminar for European Sanitary Fngineers, Rome, 11-17 November 1951. Pp. v +280. (Geneva : World Health Organization, 1952, ) 